Review Paper

\title{
Applications of Electrical Impedance Tomography in Neurology
}

\author{
Mehri Mirhoseini ${ }^{1}$ (D), Zahra Rezanejad Gatabi ${ }^{2}$ (D), Sayantan Das ${ }^{3}$ (D), Sepideh Joveini ${ }^{2}$ (D), Iman Rezanezhad Gatabi ${ }^{4 *}$ (iD) \\ 1. Amol Faculty of Paramedical Sciences, Mazandaran University of Medical Sciences, Sari, Iran. \\ 2. Department of Pharmaceutics, Faculty of Pharmacy, Mazandaran University of Medical Sciences, Sari, Iran. \\ 3. Faculty/College of Science and Mathematics, Texas A\&M University, San Antonio, United States. \\ 4. Department of Electrical and Computer Engineering, Texas A\&M University, College Station, United States.
}

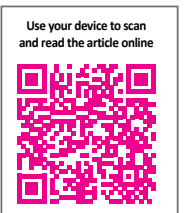

Citation Mirhoseini, M., Rezanejad Gatabi, Z., Das, S., Joveini, S., Rezanezhad Gatabi, I. (2022) Applications of Electrical Impedance Tomography in Neurology. Basic and Clinical Neuroscience, 13(5), 595-608. http://dx.doi. org $/ 10.32598 / \mathrm{bcn} .2021 .3087 .1$

oil http://dx.doi.org/10.32598/bcn.2021.3087.1

(c) (1) (s)

Article info:

Received: 30 Nov 2020

First Revision: 24 Jan 2021

Accepted: 14 May 2021

Available Online: 01 Sep 2022

Keywords:

Neurology, Electrical impedance tomography, Medical application, Brain imaging

\section{AB S T RAC T}

Introduction: Electrical impedance tomography (EIT) is a non-invasive technique utilized in various medical applications, including brain imaging and other neurological diseases. Recognizing the physiological and anatomical characteristics of organs based on their electrical properties is one of the main applications of EIT, as each variety of tissue structure has its own electrical characteristics. The high potential of brain EIT is established in real-time supervision and early recognition of cerebral brain infarction, hemorrhage, and other diseases. In this paper, we review the studies on the neurological applications of EIT.

Methods: EIT calculates the internal electrical conductivity distribution of an organ by measuring its surface impedance. A series of electrodes are placed on the surface of the target tissue, and small alternating currents are injected. The related voltages are then observed and analyzed. The electrical permittivity and conductivity distributions inside the tissue are reconstructed by measuring the electrode voltages.

Results: The electrical characteristic of biological tissues is remarkably dependent on their structures. Some tissues are better electrical conductors than the others since they have more ions that can carry the electrical charges. This difference is attributed to changes in cellular water content, membrane properties, and destruction of tight junctions within cell membranes.

Conclusion: EIT is an extremely practical device for brain imaging, capturing fast electrical activities in the brain, imaging epileptic seizures, detecting intracranial bleeding, detecting cerebral edema, and diagnosing stroke.

\section{* Corresponding Author:}




\section{Highlights}

- Electrical impedance tomography (EIT) is a new method for medical imaging.

- EIT characterizes the physiological and anatomical characteristics of organs.

- EIT is applicable in brain imaging, and imaging epileptic seizures.

\section{Plain Language Summary}

There are several techniques available for developing tissue images such as X-ray, X-ray computerized tomography (CT scanning), radio-isotope imaging, and magnetic resonance imaging (MRI). Electrical impedance tomography (EIT) is a new method of medical imaging. EIT employs multiple surface electrodes to measure the electrical impedance. The impedance is measured by injection of excitation current between two electrodes and measuring the resultant induced voltage from other electrodes. The image is then reconstructed from the measured voltages. Considering this technique's cost-effectiveness and non-invasive measurement capabilities, it has numerous medical applications such as brain imaging. EIT's measurement accuracy and its capability to detect pathological human tissues relies on two underlying facts: (a) significant impedance difference between various types of human body tissues and (b) the typical impedance difference between healthy and pathological tissues, detected by evaluating electrical distribution and its resistance variations. Although numerous studies were focused on investigating the possibility of using electrical impedance tomography for monitoring the brain, there is still a crucial requirement for efficient and non-invasive diagnosis tools in electrical bio impedance applications for monitoring the brain. In this paper, we reviewed the neurological applications of EIT and previous studies performed on this matter.

\section{Introduction}

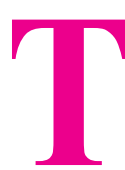

he second most common worldwide cause of death is brain damage. Early diagnosis and treatment can prevent the adverse effects of most cases of brain damage. It has been more than five decades since the electrical impedance tomography (EIT) examinations were first utilized in neurology. EIT was used to perform surface measurements to reconstruct the conductivity distribution of a cross-section. This is performed by passing a fixed current through some electrodes placed on the target surface. The fixed current induces a change in the voltages across the topography of the surface (Figure 1) which is primarily utilized to identify the inherent conductivity variations (Bayford, 2006; Packham et al., 2016).

Considering that this technique is cost-effective, noninvasive, safe, and portable, it has numerous medical applications, including evaluating gastrointestinal state, abdominal bleeding, detecting breast cancer, neuroimaging, and analyzing the blood flow of the heart, pulmonary emboli, and ventilation (Borcea, 2002).
Although the EIT technique provides an appropriate temporal resolution (Meena et al., 2018), it has a lower imaging resolution compared to magnetic resonance imaging (MRI) and computed tomography (CT) scans. Many efforts have been made to improve the quality of reconstructed images from the measured data.

These reports include EIT electrode modification (Gatabi et al., 2020; Gatabi et al.) and novel methods to determine the boundary conditions (Ren et al., 2018a; Ren et al., 2018b).

EIT measurements' accuracy and capability to detect pathological human tissues rely on two underlying facts: (a) significant impedance difference between various types of human body tissues, enabling anatomical images for a cross-section of the body, and (b) the typical impedance difference between healthy and pathological tissues, detected by evaluating electrical distribution and its resistance variations (Holder, 2005).

Since the 1950s, EIT examinations were carried out in neurology to study the influence of various physiopathological and etiology mechanisms, including seizure activity, cardiac arrest, spread depression, and asphyxia (Van et al., 1963; Van Harreveld and Ochs, 1956 , 1957; Van Harreveld and Schadé, 1962). Various bio impedance studies and clinical examinations have been 
done in the fields of perinatal asphyxia (Lingwood et al., 2002; Lingwood et al., 2003; Seoane et al., 2005), brain ischemia (Holder, 1992; Williams et al., 1991), stroke (Bonmassar and Iwaki, 2004; Liu et al., 2006; Liu et al., 2005), monitoring brain functions (Tidswell et al., 2001), epilepsy (Cusick et al., 1994; Olsson et al., 2006; Rao, 2000), and blood flow monitoring (Bodo, et al., 2004; Bodo et al., 2005; Bodo et al., 2003).

Although numerous studies were focused on investigating the possibility of using electrical impedance tomography to monitor the brain, especially in the last two decades, there is still a crucial need for efficient and non-invasive diagnosis aid tools in electrical bioimpedance applications for monitoring of the brain (Seoane Martínez, 2007). For these reasons, it is required to accumulate the current information about EIT and its application in neurology to improve this tool. Figure 2 illustrates the neurological applications of EIT.

\section{Search strategy}

A literature study was performed on key databases (PubMed, Science Direct, Scopus, and Google scholar), using the terms "Electrical Impedance Tomography (EIT)," "Detection," and "Neurology." The articles that we found were published in English and had a wide timeframe (from 1950 to 2020).

In this review, we first briefly describe the electrical properties of body tissues. Then, we describe the applications of EIT in neuroscience. Finally, we explain the applications of EIT in imaging the brain, imaging of fast electrical activities in the brain, imaging epileptic seizures, detecting intracranial bleeding, detecting cerebral edema, and diagnosing stroke.

\section{Tissue properties}

Recognizing the physiological and anatomical characteristics of organs based on their electrical properties is one of the main applications of EIT, as each variety of tissue structure has individual electrical characteristics. For example, some of the tissues can have higher electrical conductivities because of the higher availability of ions, which are considered charge carriers (Brown, 2003). Dielectric conductivity of intracranial and scalp tissues is provided in Table 1.

The tissue of the human body includes cells with fluids around them. The membrane surrounds the intracellular fluid in each cell, and the availability of electrolytes and water contents in extracellular and intracellular fluids makes them electrically resistive. Moreover, lipid bilayers with leaky ion channels that are contained in the membrane induce a capacitive and resistive behavior. The net electrical impedance of the tissue that has both capacitance and resistance can be calculated using Equation 1:

\section{1. $Z=R+j X$}

Where $\mathrm{Z}, \mathrm{R}$, and $\mathrm{X}$ indicate impedance, resistance, and reactance, respectively. The Equation 1 can be modified as Equation 2:

\section{2. $Y=G+j \omega C$}

The inverse of the impedance $(\mathrm{Z})$ is the admittance $\mathrm{Y}$, and $\mathrm{G}, \mathrm{C}$, and $\omega$ are conductance, capacitance, and angular frequency, respectively. Further modification of the above equation representing the admittance of tissue can be done as follows (Equation 3):

\section{3. $\sigma^{*}=\sigma+j \omega \varepsilon_{0} \dot{\varepsilon}$}

Where $\sigma^{*}, \dot{\varepsilon}$, and $\sigma$ represent admittance, conductivity, and permittivity of the tissue, while $\varepsilon 0$ represents the free space dielectric constant (Stojadinovic et al., 2005; Zou and Guo, 2003).

The applied electric field frequency alters the electrical properties of the tissue. Three different dispersion regions exist in tissues: low-range $\alpha$, mid-range $\beta$, and high-frequency $\gamma$. The $\alpha$-dispersion driven by the phenomena of interfacial polarization, because of the ions flowing across the membranes in the cell and the electrodes-fluid interactions, are observed in frequencies lower than $1 \mathrm{kHz}$. The $\beta$ dispersion predominantly occurs because of the plasma membranes, proteins, and other organic macromolecule polarizations, observed in several hundred $\mathrm{kHz}$ frequency ranges. The $\gamma$ dispersion contributed from the polar water molecule relaxation is observed in the frequency range of $100 \mathrm{MHz}$ and higher. Most of the differences between pathologic and normal tissues take place in the frequency ranges within $\alpha$ and $\beta$ (Gabriel et al., 2009; Stojadinovic et al., 2005; Zou and Guo, 2003).

\section{Brain imaging}

The high potential of brain EIT in real-time supervision and early recognition of cerebral brain infarction, hemorrhage, and other diseases is established. Furthermore, EIT is used for detecting nerve activities and functions. The presence of cerebrospinal fluid (CSF), 


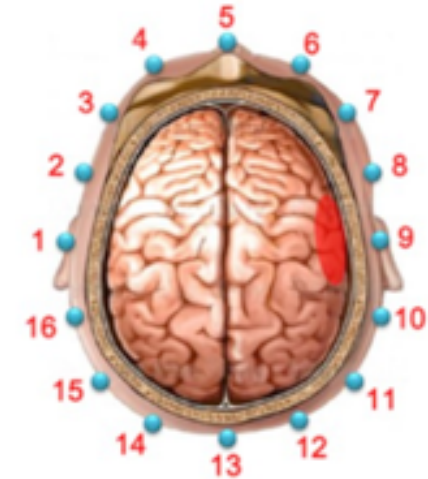

Placing electrodes on the body

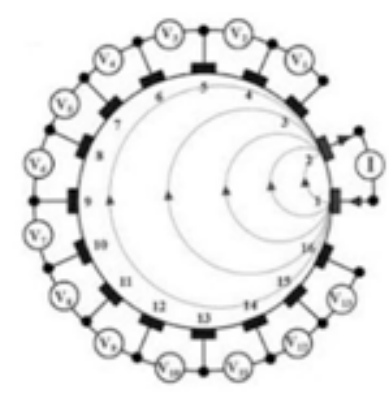

Current injection and voltage measurement

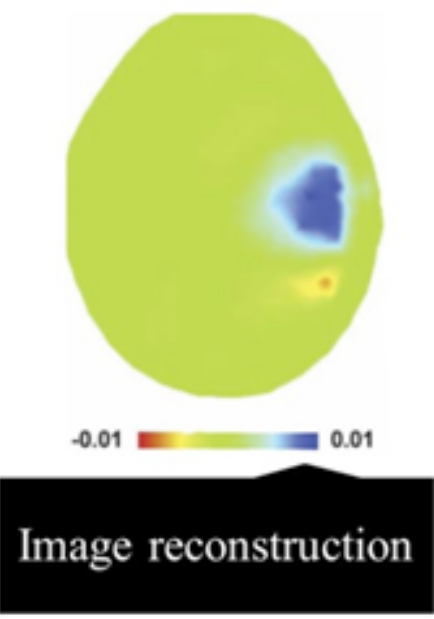

NEUR SCIENCE

Figure 1. General illustration of electrical bio impedance system (Dai e. al., 2013)

which has low resistivity and highly resistive skull adjacent to the brain tissues, makes EIT brain imaging more challenging compared to the imaging of any other body organs (Tang et al., 2010; Tehrani et al., 2010). Throughout the imaging process, the highly resistive skull tissues interfere with the measurement by being an obstacle for the measurement current to penetrate the skull. Consequently, compared to peripheral current density, a considerably lower current density can be observed in the brain. Thus, the sensitivity to internal alternations is lower in the boundary voltages, which makes it challenging to acquire data and reconstruct the appropriate image. It is recommended that a system with high accuracy data acquisition capability can be useful for the application of EIT in brain imaging (Shi et al., 2006; Shi et al., 2018).

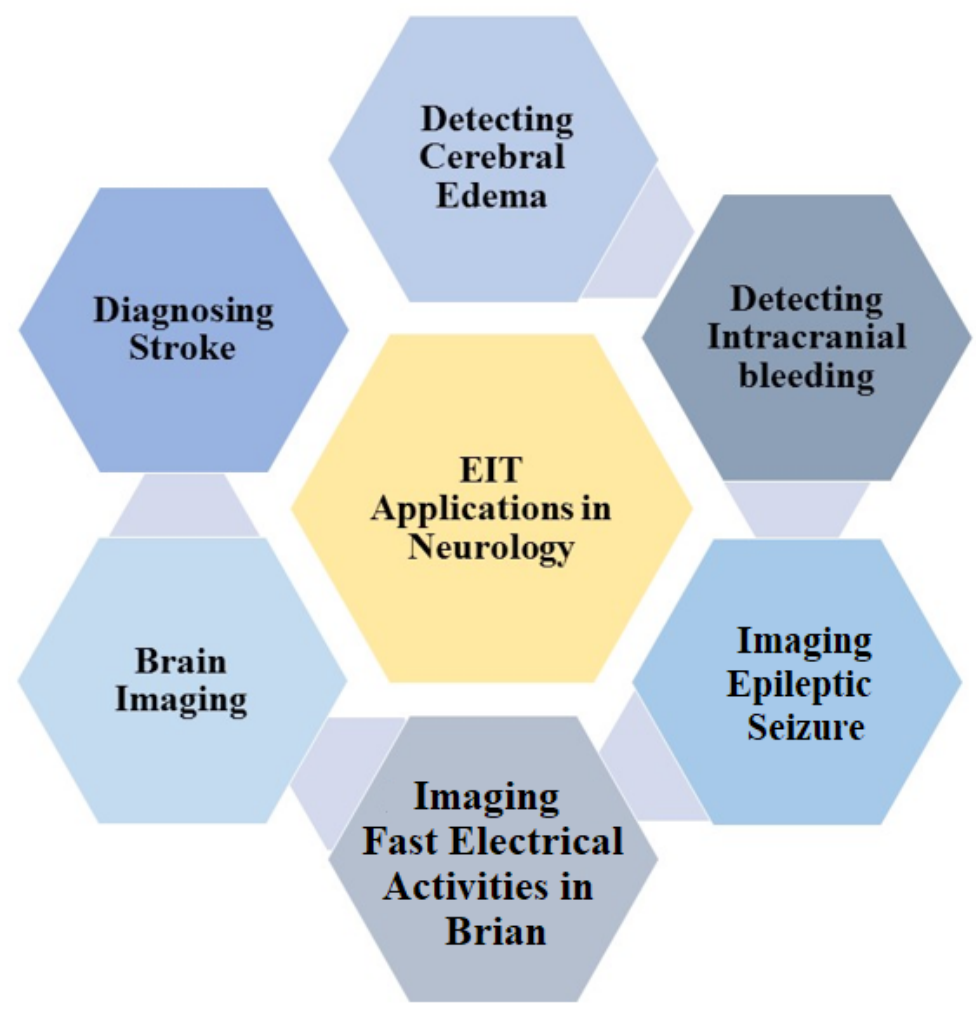

Figure 2. Applications of electrical bio impedance in neurology 
Table 1. Dielectric conductivity of intracranial and scalp tissues (Fernando, 2007)

\begin{tabular}{|c|c|c|c|}
\hline \multirow{2}{*}{ Tissue } & \multicolumn{3}{|c|}{ Conductivity $\sigma[\mathrm{S} / \mathrm{m}]$} \\
\hline & $50 \mathrm{~Hz}$ & $50 \mathrm{KHz}$ & $500 \mathrm{KHz}$ \\
\hline Blood & 0.7 & 0.7 & 0.75 \\
\hline Blood vessel & 0.26 & 0.32 & 0.32 \\
\hline Body fluid & 1.5 & 1.5 & 1.5 \\
\hline Bone cancellous & 0.08 & 0.08 & 0.087 \\
\hline Bone cortical & 0.02 & 0.021 & 0.022 \\
\hline Bone marrow & 0.0016 & 0.0031 & 0.0038 \\
\hline Cartilage & 0.17 & 0.18 & 0.21 \\
\hline Cerebellum & 0.095 & 0.15 & 0.17 \\
\hline Cerebrospinal fluid & 2 & 2 & 2 \\
\hline Fat & 0.02 & 0.024 & 0.025 \\
\hline Glands & 0.52 & 0.53 & 0.56 \\
\hline Gray matter & 0.075 & 0.13 & 0.15 \\
\hline Ligaments & 0.27 & 0.39 & 0.39 \\
\hline Lymph & 0.52 & 0.53 & 0.56 \\
\hline Muscle & 0.23 & 0.35 & 0.45 \\
\hline Nerve spine & 0.027 & 0.069 & 0.11 \\
\hline Skin dermis (dry) & 0.0002 & 0.00027 & 0.044 \\
\hline White matter & 0.053 & 0.078 & 0.095 \\
\hline
\end{tabular}

NEUR SCIENCE

\section{Imaging Fast Electrical Activities in the Brain}

Energy and oxygen consumption increase while the brain functions and consequently, the blood flow elevates. The local impedance changes as a result of the brain's physiological activities are categorized into two groups. Firstly, the gradual/slow impedance changes which happen in tens of seconds, similar to the changes in diffusion-weighted MRI or functional magnetic resonance imaging (fMRI), where the current almost entirely passes through extracellular space, enabling the impedance to rise by tens of percent. It is to be noted that the temperature, volume, and blood flow alternations, which occur during an activity, reduce impedance by a few percent. Secondly, rapid impedance changes happen in milliseconds because of the neuronal depolarization in the brain as a result of the opening of the ion channels, hence reducing the brain bulk resistance (Holder, 2008). Nowadays, imaging depolarization of neuronal cell bodies and their processing has attracted much attention. In the field of neuroscience, imaging the brain neuronal depolarization is very important and has garnered much interest among researchers. However, a method that could image the whole neural activity in the brain over milliseconds is not available yet.

EIT can potentially image the impedance variations that occur throughout rapid electrical activities as a result of neuronal depolarization. As neurons are activated, the voltage- and ligand-gated ion channels in neuronal cell membranes open up and permit the electrical current to transit through the intracellular space. As a result, it alters the voltages on recording electrodes of the EIT and it was tested on rats after anesthesia by utilizing an epicortical electrode array. This 
helped analyze the cortical neural activities throughout a somatosensory that evoked the potential with spatial and temporal resolutions of less than $2 \mathrm{~ms}$ and $200 \mu \mathrm{m}$, respectively. The depth of penetration was limited to 2 $\mathrm{mm}$; hence, the impedance variations sensitivity was found to be limited to the cerebral cortex. A simulation study (Aristovich et al., 2016) indicated that by implementing some enhanced experimental protocols, imaging the rapid electrical activities in the rat brain was possible with less than $1 \mathrm{~mm}$ localization. For a 1-mm localization accuracy, application of rapid variations in impedance because of individual epileptic discharges can be employed and during these discharges, calcium, and sodium ion channels open up, which are dependent on the voltage, enabling hypersynchronous depolarization of the local neuronal populations in the brain (Hannan et al., 2018b; Stafstrom, 2007).

\section{Imaging Epileptic Seizure}

Among the neurological disorders, epilepsy is found to be the second most common, affecting 60 million people worldwide. For approximately $75 \%$ of these cases, the use of suitable anticonvulsant drugs can either stop or reduce seizures. Neurosurgery can help $7 \%$ to $8 \%$ of the patients by surgically removing the source of the seizure from their brain (Hannan et al., 2018b; Wang et al., 2017b). The success rate of the operation of epilepsy originating from a unique focus is severely dependent on the accuracy of locating the seizure source and for about $25 \%$ of these cases, seizures cannot be relieved by surgery alone. These failures arise because of an inaccurate recognition of the focus or the existence of several or diffuse foci (Fabrizi et al., 2006). It is to be noted that the images obtained showing diffuse or numerous lesions make it inadvisable to perform refractory epilepsy surgery (Spencer and Huh, 2008). Different imaging capabilities are employed to recognize epileptogenic lesions, including positron emission tomography (PET), CT, and MRI. Although each of these methods has some restrictions, MRI and CT can discover abnormal structures but cannot locate the functional lesions. CT scan alone presents a considerably low sensitivity in the recognition of usual brain pathologies. Meanwhile, the requirements of using scanners in PET make it unusable for continuous monitoring. Single-photon emission computed tomography (SPECT) is extensively utilized to measure regional cerebral blood flow ( $\mathrm{rCBF}$ ). An auxiliary method, similar to SPECT, is suitable for the pre-surgery examination of patients with intractable focal seizures (O’brien et al., 1998). However, this method utilizes radioisotopes that are injected following the seizure onset, and the rCBF associated with seizures in- creases with a delay (La Fougère et al., 2009). Hence, an enhanced imaging method is required for pre-surgery examinations of patients with refractory epilepsy; accordingly, EIT can be employed.

EIT imaging technique reconstructs 3D images of differences in internal conductivities of an object based on boundary evaluations (Witkowska-Wrobel et al., 2018) and can be used for identifying epileptic foci during presurgical examinations of neurosurgery. To use EIT for this purpose, the presumed ictal onset zone is resected to control the seizures (Fabrizi et al., 2006; Hannan et al., 2018a). Throughout the seizure, by applying 1 to 50 $\mathrm{kHz}$ of current, the electrical conductivity of some locations in the brain reduces by almost $3 \%$ to $12 \%$ (Fabrizi et al., 2010).

EIT has the potential to locate the epileptogenic region more accurately by using local impedance. Two different forms of variations in the local impedance can occur during seizure activities. The first is the slow variations in impedance which are found to happen more than $10 \mathrm{~s}$ after the seizure onset and have a magnitude of up to $12 \%$ (Wang et al., 2017b). The cell swelling and extracellular space shrinkage throughout the seizures are the reasons for these impedance variations, and they result in changes in extracellular water and potassium ions because of considerable depolarization in the region (Niermann, $\mathrm{H}$ et al., 2001). The second is the fast variations in impedance and recent studies show that these variations have a magnitude up to $1 \%$ (Vongerichten et al., 2016) which occurs because of the variations that can be traced back to the ion channels opening throughout synchronized neuronal depolarization (Oh et al., 2011).

\section{Detecting Intracranial Bleeding}

Rupture or leakage of blood vessels in the skull causes hemorrhage or intracranial bleeding, and as a result, blood accumulates inside the cranial vault. This process can be spontaneous and can be caused by an underlying vascular malformation that arises from therapeutic anticoagulation, or trauma. The increased intracranial pressure (ICP) can limit the brain blood supply or crush sensitive tissues of the brain and result in possibly fatal brain herniation. Intracerebral hemorrhage (ICH) is among the most severe emergencies in the medical field. In acute conditions, to reduce swelling and stop the bleeding, a surgical operation should be performed (Dai et al., 2013). Currently, there are various difficulties to diagnose and treat secondary $\mathrm{ICH}$. 
After cranial surgery, postoperative CT scans are utilized to examine the success of the surgery and identify the difficulties (Xu et al., 2010). The problem with a CT scan is that it can only produce an image at a specific time after the hematoma formation, and it cannot be employed for continuous monitoring. Thus, the CT scan is usually incapable of recognizing secondary ICH. For this reason, the development of a radiationfree and non-invasive method that can detect the onset of intracranial bleeding and monitor it continually is crucial (Dai et al., 2013; Xu et al., 2010).

EIT is a radiation-free and non-invasive imaging method and can present promising diagnostic data regarding ICH (Yang et al., 2014). As the impedance of brain tissues and blood are quite different, when the blood leaks into the brain tissues, it considerably alters the impedance distribution. These changes can be monitored utilizing EIT and studies reveal that intraperitoneal and retroperitoneal bleedings affect the impedance variations; therefore, EIT can be used for monitoring these issues (Shuai et al., 2009; Wanjun et al., 2008). Some reports state that bleeding in the brain increases the electrical impedance of the brain (Dai et al., 2013; Dai et al., 2010), while some other studies show that intracerebral hemorrhage reduces the electrical impedance of the brain (Manwaring et al., 2013; Wang et al., 2016).

In a study conducted by $\mathrm{Xu}$ et al. (2010), they utilized EIT for detecting ICH in piglet models, where blood was injected into the brain's frontal lobe of the anesthetized animals and the electrical impedance was recorded utilizing a series of electrodes which were located on the scalp. Finally, EIT images were produced by utilizing a computer algorithm (filtered back-projection). To measure the relative impedance variations, the average resistivity value (ARV) was examined for the target zone, and the asymmetrical index (SI), which shows the relative impedances for both sides of the brain, was computed. Alterations in these two values because of the blood injection indicated that EIT could be considered a useful tool for recognizing $\mathrm{ICH}$ in animal models.

In another investigation by Wang et al. (2017a), blood was injected into pig models to create an $\mathrm{ICH}$, where both ARV and electrical impedance was employed to measure the variations in electrical impedance because of the blood injection. ARV values show the variations in electrical impedance of the target zone, while EIT indicates the electrical impedance variations for the whole brain. The findings suggested that EIT and ARV values were reduced after the blood injection. These two values for the experimental group were significantly lower compared to the control group $(\mathrm{P}<0.01)$. The results indicate that EIT can be affected by $\mathrm{ICH}$ in animals; thus, this method may be an excellent tool for monitoring $\mathrm{ICH}$.

\section{Detecting cerebral edema}

Cerebral edema is a common complication as a result of different disorders in the nervous system, such as brain trauma, cerebral stroke, and other craniocerebral disorders. With this fatal disease, considerable brain damage or even death is probable (Wang et al., 2017a). When a patient has this clinical condition, excess fluid accumulates in the extracellular or intracellular brain spaces. Cerebral edema is a frequent neurological emergency state, and its formation results in high morbidity and mortality in the patient ( $\mathrm{Fu}$ et al., 2014). Monitoring the brain edema and early diagnosis can significantly help the treatment and predict the neurological result along with inhibiting more damage. Currently, a method for continuous monitoring of brain edema because of cerebral infarction with adequate accuracy does not exist. CT scan and MRI are expensive tools used to identify brain edema; however, these methods cannot provide continuous monitoring of brain edema. Also, they are incapable of showing real-time impacts of the treatment (Liu et al., 2006; Liu et al., 2005). ICP monitoring is another method for diagnosing cerebral edema which can indirectly illustrate craniocerebral injury advancement. It can perform continuous monitoring of cerebral edema. However, ICP is expensive, invasive, and not suitable for clinical practices. Therefore, the development of a non-invasive method for monitoring cerebral edema in real time is crucial (Padayachy, 2016).

EIT is an inexpensive, reliable, non-invasive technique where patients do not experience any discomfort or risk, and the equipment is portable and can be utilized for continuous monitoring (Liu et al., 2006). Since normal and cerebral edema tissue impedances differ in value, electrically conductive physiological fluids alter the brain tissue impedance variations and consequently allow the EIT to provide continuous image monitoring for brain edema. For identifying cerebral edema using EIT, the electrical impedance attributes of cerebral edema tissues should be accurately evaluated (Fu et al., 2014). 
In a study conducted by Song et al. (Song et al., 2018), cerebral edema in a rat model was examined. After the descent of ischemic brain injury, electrical impedance changes in different points of cerebral edema and associated morphologic changes were monitored for 24 h. Six hours later at the descent of ischemic brain injury, the brain tissue's electrical resistivity was raised significantly $(\mathrm{P}<0.05)$. Throughout this entire period, the volume of the brain cell expanded significantly ( $\mathrm{P}$ $<0.05$ ); however, the intercellular space was significantly reduced $(\mathrm{P}<0.05)$. For the next 6 - to $24-\mathrm{h}$ period, the brain tissue's electrical resistivity lowered and the volume of the brain cell was constant $(\mathrm{P}>0.05)$; even though the intercellular space was significantly expanded $(\mathrm{P}<0.05)$. This study suggested that EIT can be utilized for continuous monitoring of the advancement of cerebral edema.

He et al. (He et al., 2010) used cerebral electrical impedance (CEI) evaluation to examine the brain edema changes among patients with cerebral infarction. The bilateral hemisphere CEI values from 200 healthy volunteers and 107 cerebral infarction patients were evaluated. These were used to calculate the perturbative index (PI) values. The calculated PI values were further compared to the calculated brain edema volumes based on the MRI and CT scan imaging techniques. The PI values of healthy volunteers for the right and left hemispheres were $8.02 \pm 0.71$ and $7.98 \pm 0.95$, respectively, with no significant difference between the right and the left hemisphere's PI values $(\mathrm{P}>0.05)$. Also, gender, age, and period of evaluation did not have any significant impact on the PI values $(\mathrm{P}>0.05)$. Among the cerebral infarction patients, the sensitivity of CEI evaluations for lesions larger than $20 \mathrm{~mL}$ volume was higher, with the PI ratio as high as $80.0 \%$. For the infarction volume ranging from 20 to $50 \mathrm{~mL}$ a PI ratio of $75.9 \%$ was presented, while for lesions with volumes higher than $50 \mathrm{~mL}$, it was $83.3 \%$. It is to be noted that lesions smaller than $20 \mathrm{~mL}$ had a much lower PI value. Among the cerebral infarction patients, the difference between the two hemispheres was considerable, and a positive correlation was observed between the infarction side PI and the infarction volume. Overall, PI was a sensitive parameter for brain edema monitoring in patients who have cerebral infarction, and CEI was considered a useful technique for the earlier recognition of brain edema.

\section{Diagnosing stroke}

Acute stroke, which is also known as severe cerebrovascular disease, is one of the most common causes of death worldwide after heart and cancer diseases (Lingwood et al., 2003). Acute strokes can be categorized into two varieties. The first is hemorrhagic stroke, accounting for almost $13 \%$ of strokes. This occurs because of bleeding into the subarachnoid space or brain tissues due to a rupture in an intracranial vessel. The second is ischemic stroke, accounting for almost $87 \%$ of strokes and it occurs because of the thrombosis in brain vessels which results in vascular occlusion in the brain (Bamford et al., 1991). Even though the probability of hemorrhagic strokes is lower compared to ischemic strokes, the possibility of death is much higher in the former. To manage strokes and initiate the proper treatment, the first step is to determine the type of stroke which is an important challenge (Donnan et al., 2009). The treatments for these two types of strokes are quite different. For instance, thrombolytic which is the treatment for ischemic stroke can be harmful, or even fatal, for patients who have had a hemorrhagic stroke. Therefore, it is important to use neuroimaging techniques to determine the type of strokes in patients before starting any treatments. On the other hand, better results from the treatments can be obtained if early treatments are executed within the first $6 \mathrm{~h}$ of the stroke (Hacke et al., 2008; Stemer and Lyden, 2010).

The current neuroimaging techniques, such as MRI or CT scan cannot be employed for rapid stroke diagnosis since preparing an image and presenting a report based on these techniques usually take at least $1 \mathrm{~h}$. Also, these imaging techniques are typically not available in ambulances or casualty departments (Yang et al., 2016a). Without a rapid neuroimaging technique, patients cannot receive accurate diagnostics and critical treatment on time. EIT is an affordable, harmless, and portable alternative imaging technique that is capable of producing images based on the internal electrical conductivity of the target.

The electrical impedance of the ischemic tissues is abnormally high, and the cell swelling is attributed to this high impedance because it results in a smaller extracellular space and raises the impedance of the bulk tissue to $50 \%$ (Holder, 1992). EIT can also be used to detect hemorrhagic strokes where the brain electrical conductivity $(\sigma \mathrm{h}=0.15 \mathrm{~S} / \mathrm{m})$ is almost $75 \%$ lower than the blood electrical conductivity $(\sigma \mathrm{h}=0.61 \mathrm{~S} / \mathrm{m})$ (Clay \& Ferree, 2002). 
Differences in the electrical conductivities of the blood and healthy and ischemic brain tissues ( $\Omega$ blood $<\Omega$ brain $<$ Sischemia) have been revealed experimentally. EIT uses these differences in electrical conductivities (Dowrick et al., 2015) for its measurements. The EIT equipment is economical and portable, can be available in ambulances or general practitioner surgeries, and rapidly present images that can greatly improve the thrombolytic management of the stroke.

In most of the studies which have focused on the applications of EIT in stroke, the tests are performed on animal models. Shi et al. (Shi et al., 2008) simulated ischemia in rabbit models by inducing ischemia in the rabbits where a hole was drilled in their skull, and their brain was exposed to direct irradiation through the hole. The data indicating the alterations in the electrical impedance was collected by utilizing a series of electrodes located on the skull, and the results indicated a rapid increase in the electrical impedance. Both scalp and cortical electrodes were successfully used for producing images of global ischemia in rat models (Dowrick et al., 2016; Holder, 1992). The UCH Mk2.5 EIT system was employed to diagnose strokes in humans (Romsauerova et al., 2006). Although the collected data was robust, the quality of the produced images was not enough to make them practical for clinical use.

In the investigation conducted by Yang et al. (Yang et al., 2017), the hemorrhagic and ischemic conditions were modeled among rabbits. Brain's electrical impedance spectra under healthy, hemorrhagic, and ischemic conditions were evaluated in vivo with frequencies ranging from $10 \mathrm{~Hz}$ and $1 \mathrm{MHz}$. Then, to analyze the possibility of employing EIT for diagnosing stroke, impedance spectra differences between the brains which were healthy or affected by a stroke were compared. Also, for differentiating ischemic and hemorrhagic strokes and determining the stroke type, the rates of brain impedance variations concerning frequency were compared. The results indicated the difference between impedance spectra of the brains which were healthy or affected by either of these two kinds of strokes, were highly distinguishable in the range between $10 \mathrm{~Hz}$ and $1 \mathrm{MHz}$. Also, the difference between the real part of electrical impedance in a stroke-affected versus a healthy brain was as high as $6 \Omega$. This contrast also existed when the brain impedance rate of brains with ischemic and hemorrhagic stroke conditions were compared in terms of frequency. In the range of $500 \mathrm{kHz}-1 \mathrm{MHz}$, the variations in the imaginary part of brain impedance for the ischemic brain were almost 3 times lower than those of the brain with the hemorrhagic condition.

In an examination performed by Yang et al. (Yang et al., 2016b), ischemic and intracerebral hemorrhage conditions in rabbit models were simulated. They evaluated the electrical impedance spectra for brain tissues, which were healthy or affected by stroke ex vivo. The evaluations were performed within $15 \mathrm{~min}$ after the death of the animal models in the frequency range of $10 \mathrm{~Hz}$ and $1 \mathrm{MHz}$. According to their findings, the standard brain tissue impedance spectra were considerably different from the impedance spectra of the stroke lesions (for the frequency ranging from $10 \mathrm{~Hz}$ to $1 \mathrm{MHz}$, there was more than a $10 \%$ difference between the electrical conductivity of stroke lesions and the healthy brain tissue). They also stated that the impedance variation ratio in tissues with hemorrhagic and ischemic conditions in terms of frequency was significantly different. Based on these electrical impedance differences, the type of tissue could be determined.

\section{Conclusion}

EIT is a simple, non-invasive, and economical technique. Thus, it can be considered an attractive research field in the near future. Since the spatial resolution of EIT cannot be compared with CT scans and MRI, it requires more qualitative studies. Several studies have been performed on the EIT system, with most of the investigations focusing on the applications of the EIT system on animal models. In these studies, electrical conductivity was used to evaluate electrical resistivity parameters in tissues. The differences in the electrical resistivity of healthy and affected tissue can be used to distinguish them from each other.

For future studies, it would be beneficial to investigate the application of EIT for real-time monitoring of patients under operation, especially for brain surgery, to differentiate cancerous tissues from healthy tissues. If such an application is feasible, it will eliminate the need for pathological tissue sampling and waiting for pathological lab results for subsequent surgery to extract the cancerous tissues. The EIT method has significant diagnostic capabilities. However, it does not have a high resolution. Future researchers may also focus on ways to increase the resolution of the EIT method, for example by implementing image reconstruction algorithms or improving the active surface areas of the electrodes by using nanoparticles. 


\section{Ethical Considerations}

\section{Compliance with ethical guidelines}

There were no ethical considerations to be considered in this research

\section{Funding}

This research did not receive any grant from funding agencies in the public, commercial, or non-profit sectors.

\section{Authors' contributions}

All authors equally contributed to preparing this article.

\section{Conflict of interest}

The authors declared no conflict of interest.

\section{Acknowledgments}

We wish to acknowledge the conditions provided by Mazandaran University of Medical Sciences, Sari, Iran for providing the research opportunities.

\section{References}

Aristovich, K. Y., Packham, B. C., Koo, H., Santos, G., McEvoy, A., \& Holder, D. S. (2016). Imaging fast electrical activity in the brain with electrical impedance tomography. NeuroImage, 124(Pt A), 204-213. [PMID] [PMCID]

Bamford, J., Sandercock, P., Dennis, M., Burn, J., \& Warlow, C. (1991). Classification and natural history of clinically identifiable subtypes of cerebral infarction. Lancet (London, England), 337(8756), 1521-1526. [DOI:10.1016/0140-6736(91)93206-O]

Bayford R. H. (2006). Bioimpedance tomography (electrical impedance tomography). Annual Review of Biomedical Engineering, 8, 63-91.[DOI:10.1146/annurev.bioeng.8.061505.095716] [PMID]

Bodo, M., Pearce, F. J., \& Armonda, R. A. (2004). Cerebrovascular reactivity: Rat Studies in rheoencephalography. Physiological Measurement, 25(6), 1371-1384. [PMID]

Bodo, M., Pearce, F. J., Baranyi, L., \& Armonda, R. A (2005). Changes in the intracranial rheoencephalogram at lower limit of cerebral blood flow autoregulation. Physiological Measurement, 26(2), S1-S17. [DOI:10.1088/09673334/26/2/001] [PMID]

Bodo, M., Pearce, F. J., Montgomery, L. D., Rosenthal, M. Kubinyi, G., \& Thuroczy, G., et al. (2003). Measurement of brain electrical impedance: Animal studies in rheoencepha- lography. Aviation, Space, and Environmental Medicine, 74(5), 506-511. [PMID]

Bonmassar, G., \& Iwaki, S. (2004). The shape of electrical impedance spectroscopy (EIS) is altered in stroke patients. Conference proceedings : Annual International Conference of the IEEE Engineering in Medicine and Biology Society. IEEE Engineering in Medicine and Biology Society. Annual Conference, 2004, 3443-3446. [PMID]

Borcea, L. (2002). Electrical impedance tomography. Inverse Problems, 18(6), R99. [DOI:10.1088/0266-5611/18/6/201]

Brown B. H. (2003). Electrical impedance tomography (EIT): A review. Journal of Medical Engineering E Technology, 27(3), 97-108. [PMID]

Clay, M. T., \& Ferree, T. C. (2002). Weighted regularization in electrical impedance tomography with applications to acute cerebral stroke. IEEE Transactions on Medical Imaging, 21(6), 629-637. [DOI:10.1109/TMI.2002.800572] [PMID]

Cusick, G., Holder, D. S., Birkett, A., \& Boone, K. (1994). A system for impedance imaging epilepsy in ambulatory human subjects. Paper Presented at CAIT MeetingSeminaire CAIT Toulouse, France, 14 March 1994. [Link]

Dai, M., Li, B., Hu, S., Xu, C., Yang, B., \& Li, J., et al.( 2013) In vivo imaging of twist drill drainage for subdural hematoma: a clinical feasibility study on electrical impedance tomography for measuring intracranial bleeding in humans. PloS one, 8(1), e55020. [PMID]

Dai, M., Wang, L., Xu, C., Li, L., Gao, G., \& Dong, X. (2010). Real-time imaging of subarachnoid hemorrhage in piglets with electrical impedance tomography. Physiological Measurement, 31(9), 1229-1239. [PMID]

Donnan, G. A., Baron, J. C., Ma, H., \& Davis, S. M. (2009) Penumbral selection of patients for trials of acute stroke therapy. The Lancet. Neurology, 8(3), 261-269. [DOI:10.1016/ S1474-4422(09)70041-9]

Dowrick, T., Blochet, C., \& Holder, D. (2015). In vivo bioimpedance measurement of healthy and ischaemic rat brain: Implications for stroke imaging using electrical impedance tomography. Physiological Measurement, 36(6), 1273-1282. [PMID]

Dowrick, T., Blochet, C., \& Holder, D. (2016). In vivo bioimpedance changes during haemorrhagic and ischaemic stroke in rats: towards 3D stroke imaging using electrical impedance tomography. Physiological Measurement, 37(6), 765-784. [PMID]

Fabrizi, L., Sparkes, M., Horesh, L., Perez-Juste Abascal, J. F. McEwan, A., \& Bayford, R. H., et al. (2006). Factors limiting the application of electrical impedance tomography for identification of regional conductivity changes using scalp electrodes during epileptic seizures in humans. Physiological Measurement, 27(5), S163-S174. [PMID]

Fabrizi, L., Yerworth, R., McEwan, A., Gilad, O., Bayford, R. \& Holder, D. S. (2010). A method for removing artefacts from continuous EEG recordings during functional electrical impedance tomography for the detection of epileptic seizures. Physiological Measurement, 31(8), S57-S72. [PMID]

Fernando, S. (2007). Electrical bioimpedance cerebral monitoring: Fundamental steps towards clinical application $[\mathrm{PhD}$ Dissertation]. Chalmers: Chalmers University of Technology and University College of Boras. [Link] 
Fu, F., Li, B., Dai, M., Hu, S. J., Li, X., \& Xu, C. H., et al. (2014), Use of electrical impedance tomography to monitor regional cerebral edema during clinical dehydration treatment. Plos One, 9(12), e113202. [PMID] [PMCID]

Gabriel, C., Peyman, A., \& Grant, E. H. (2009). Electrical conductivity of tissue at frequencies below $1 \mathrm{MHz}$. Physics in Medicine \& Biology, 54(16), 4863-4878. [PMID]

Gatabi, Z. R., Mohammadpour, R., Gatabi, J. R., Mirhoseini, M., Ahmadi, M., \& Sasanpour, P. (2020). Sandblasting improves the performance of electrodes of miniature electrical impedance tomography via double layer capacitance. Heliyon, 6(4), e03652. [PMID] [PMCID]

Rezanejad Gatabi, Z., Mohammadpour, R., Rezanejad Gatabi, J., Mirhoseini, M., \& Sasanpour, P. (2020). A novel composite gold/gold nanoparticles/carbon nanotube electrode for frequency-stable micro-electrical impedance tomography. Journal of Materials Science: Materials in Electronics, 31(13), 10803-10810. [Link]

Hacke, W., Kaste, M., Bluhmki, E., Brozman, M., Dávalos, A. \& Guidetti, D., et al. (2008). Thrombolysis with alteplase 3 to 4.5 hours after acute ischemic stroke. The New England Journal of Medicine, 359(13), 1317-1329. [PMID]

Hannan, S., Faulkner, M., Aristovich, K., Avery, J., \& Holder D. (2018). Frequency-dependent characterisation of impedance changes during epileptiform activity in a rat model of epilepsy. Physiological Measurement, 39(8), 085003. [PMID]

Hannan, S., Faulkner, M., Aristovich, K., Avery, J., Walker, M., \& Holder, D. (2018). Imaging fast electrical activity in the brain during ictal epileptiform discharges with electrical impedance tomography. NeuroImage. Clinical, 20, 674684. [PMID] [PMCID]

He, L. Y., Wang, J., Luo, Y., Dong, W. W., \& Liu, L. X. (2010). Application of non-invasive cerebral electrical impedance measurement on brain edema in patients with cerebral infarction. Neurological Research, 32(7), 770-774. [PMID]

Holder, D. S. (1992). Detection of cerebral ischaemia in the anaesthetised rat by impedance measurement with scalp electrodes: Implications for non-invasive imaging of stroke by electrical impedance tomography. Clinical Physics and Physiological Measurement, 13(1), 63-75. [PMID]

Holder, D. S. (2005). Introduction to biomedical electrical impedance tomography Electrical Impedance Tomography Methods, History and Applications ed DS Holder. Bristol: Institute of Physics.

Holder, D. S. (2008). Electrical impedance tomography of brain function. Paper presented at: Automation Congress. [Link]

la Fougère, C., Rominger, A., Förster, S., Geisler, J., \& Bartenstein, P. (2009). PET and SPECT in epilepsy: A critical review. Epilepsy $\mathcal{E}$ Behavior: EEB, 15(1), 50-55. [PMID]

Lingwood, B.E., Dunster, K.R., Colditz, P.B., Ward, L.C., 2002. Noninvasive measurement of cerebral bioimpedance for detection of cerebral edema in the neonatal piglet. Brain research 945, 97-105. [DOI:10.1016/S0006-8993(02)02744-0]

Lingwood, B. E., Dunster, K. R., Healy, G. N., Ward, L. C., \& Colditz, P. B. (2003). Cerebral impedance and neurological outcome following a mild or severe hypoxic/ischemic episode in neonatal piglets. Brain Research, 969(1-2), 160-167. [PMID]
Liu, L., Dong, W., Ji, X., Chen, L., Chen, L., \& He, W., et al. (2006). A new method of noninvasive brain-edema monitoring in stroke: cerebral electrical impedance measurement. Neurological Research, 28(1), 31-37. [PMID]

Liu, L. X., Dong, W. W., Wang, J., Wu, Q., He, W., \& Jia, Y. J. (2005). The role of noninvasive monitoring of cerebral electrical impedance in stroke. Acta Neurochirurgica. Supplement, 95, 137-140. [PMID]

Manwaring, P. K., Moodie, K. L., Hartov, A., Manwaring, K. H., \& Halter, R. J. (2013). Intracranial electrical impedance tomography: A method of continuous monitoring in an animal model of head trauma. Anesthesia and Analgesia, 117(4), 866-875. [PMID]

Meena, R. K., Pahuja, S. K., Queyam, A. B., \& Sengupta, A. (2018). Electrical impedance tomography: A real-time medical imaging technique. In I. Anwar, A. Khosla, \& R. Kapoor (eDS.), Handbook of research on advanced concepts in real-time image and video processing (pp. 130-152). Pennsylvania: IGI Global. [DOI:10.4018/978-1-5225-2848-7.ch006]

Niermann, H., Amiry-Moghaddam, M., Holthoff, K., Witte, O. W., \& Ottersen, O. P. (2001). A novel role of vasopressin in the brain: Modulation of activity-dependent water flux in the neocortex. The Journal of Neuroscience : The Official Journal of the Society for Neuroscience, 21(9), 3045-3051.[PMID]

O'Brien, T. J., So, E. L., Mullan, B. P., Hauser, M. F., Brinkmann, B. H., \& Bohnen, N. I., et al. (1998). Subtraction ictal SPECT co-registered to MRI improves clinical usefulness of SPECT in localizing the surgical seizure focus. Neurology, 50(2), 445-454. [PMID]

Oh, T., Gilad, O., Ghosh, A., Schuettler, M., \& Holder, D. S. (2011). A novel method for recording neuronal depolarization with recording at $125-825 \mathrm{~Hz}$ : Implications for imaging fast neural activity in the brain with electrical impedance tomography. Medical \& Biological Engineering \& Computing, 49(5), 593-604. [PMID]

Olsson, T., Broberg, M., Pope, K. J., Wallace, A., Mackenzie, L., \& Blomstrand, F., et al. (2006). Cell swelling, seizures and spreading depression: An impedance study. Neuroscience, 140(2), 505-515. [PMID]

Packham, B., Barnes, G., Dos Santos, G. S., Aristovich, K., Gilad, O., \& Ghosh, A., et al. (2016). Empirical validation of statistical parametric mapping for group imaging of fast neural activity using electrical impedance tomography. Physiological Measurement, 37(6), 951-967. [PMID] [PMCID]

Padayachy L. C. (2016). Non-invasive intracranial pressure assessment. Child's Nervous System : Chns : Official Journal of the International Society for Pediatric Neurosurgery, 32(9), 1587-1597. [PMID]

Rao, A. (2000). Electrical impedance tomography of brain activity: studies into its accuracy and physiological mechanisms. London: University of London. [Link]

Ren, S., Soleimani, M., Xu, Y., \& Dong, F. (2018). Inclusion boundary reconstruction and sensitivity analysis in electrical impedance tomography. Inverse Problems in Science and Engineering 26(7), 1037-1061. [DOI:10.1080/17415977.2017.1378195] 
Ren, S., Wang, Y., Liang, G., \& Dong, F.( 2018). A robust inclusion boundary reconstructor for electrical impedance tomography with geometric constraints. IEEE Transactions on Instrumentation and Measurement, 68(3), 762-773. [DOI:10.1109/TIM.2018.2853358]

Romsauerova, A., McEwan, A., Horesh, L., Yerworth, R., Bayford, R. H., \& Holder, D. S. (2006). Multi-frequency electrical impedance tomography (EIT) of the adult human head: initial findings in brain tumours, arteriovenous malformations and chronic stroke, development of an analysis method and calibration. Physiological Measurement, 27(5), S147-S161. [PMID]

Seoane, F., Lindecrantz, K., Olsson, T., Kjellmer, I., Flisberg, A., \& Bågenholm, R. (2005). Spectroscopy study of the dynamics of the transencephalic electrical impedance in the perinatal brain during hypoxia. Physiological Measurement, 26(5), 849-863. [PMID]

Seoane Martínez, F., \& Lindecrantz. (2007). Electrical bioimpedance cerebral monitoring. Göteborg: Chalmers tekniska högskola. [DOI:10.4018/978-1-59904-889-5.ch061]

Shi, X., Dong, X., Shuai, W., You, F., Fu, F., \& Liu, R. (2006). Pseudo-polar drive patterns for brain electrical impedance tomography. Physiological Measurement, 27(11), 1071-1080. [PMID]

Shi, X., Li, W., You, F., Huo, X., Xu, C., \& Ji, Z., et al. ( 2018). High-precision electrical impedance tomography data acquisition system for brain imaging. IEEE Sensors Journal, 18(14), 5974-5984. [Link]

Shi, X., You, F., Fu, F., Liu, R., You, Y., Dai, M., Dong, X., 2008. Preliminary research on monitoring of cerebral ischemia using electrical impedance tomography technique, 2008 30th Annual International Conference of the IEEE Engineering in Medicine and Biology Society. IEEE, pp. 11881191. [DOI:10.1109/iembs.2008.4649375] [PMID]

Shuai, W., You, F., Zhang, H., Zhang, W., Fu, F., \& Shi, X., et al. (2009). Application of electrical impedance tomography for continuous monitoring of retroperitoneal bleeding after blunt trauma. Annals of Biomedical Engineering 37(11), 23732379. [PMID]

Song, J., Chen, R., Yang, L., Zhang, G., Li, W.,\& Zhao, Z., et al. (2018). Electrical impedance changes at different phases of cerebral edema in rats with ischemic brain injury. BioMed Research International, 2018, 9765174. [PMID] [PMCID]

Spencer, S., \& Huh, L. (2008). Outcomes of epilepsy surgery in adults and children. The Lancet. Neurology, 7(6), 525-537. [DOI:10.1016/S1474-4422(08)70109-1]

Stafstrom C. E. (2007). Persistent sodium current and its role in epilepsy. Epilepsy Currents, 7(1), 15-22. [PMID]

Stemer, A., \& Lyden, P. (2010). Evolution of the thrombolytic treatment window for acute ischemic stroke. Current Neurology and Neuroscience Reports, 10(1), 29-33. [PMID]

Stojadinovic, A., Nissan, A., Gallimidi, Z., Lenington, S., Logan, W., \& Zuley, M., et al. (2005). Electrical impedance scanning for the early detection of breast cancer in young women Preliminary results of a multicenter prospective clinical trial. Journal of Clinical Oncology, 23(12), 2703-2715. [PMID]
Tang, T., Oh, S., \& Sadleir, R. J. (2010). A robust current pattern for the detection of intraventricular hemorrhage in neonates using electrical impedance tomography. Annals of Biomedical Engineering, 38(8), 2733-2747. [PMID]

Tehrani, J. N., Anderson, C., Jin, C., Van Schaik, A., Holder, D., \& McEwan, A. (2010). Feasibility of electrical impedance tomography in haemorrhagic stroke treatment using adaptive mesh. Journal of Physics: Conference Series, 224(1), 012065 [DOI:10.1088/1742-6596/224/1/012065]

Tidswell, A. T., Gibson, A., Bayford, R. H., \& Holder, D. S. (2001). Electrical impedance tomography of human brain activity with a two-dimensional ring of scalp electrodes. Physiological Measurement, 22(1), 167-175. [PMID]

Van Harreveld, A., Murphy, T., \& Nobel, K. W. (1963). Specific impedance of rabbit's cortical tissue. The American Journal of Physiology, 205, 203-207. [PMID]

OCHS, S., \& Van Harreveld, A. (1956). Cerebral impedance changes after circulatory arrest. The American Journal of Physiology, 187(1), 180-192. [PMID]

Van Harreveld, A., \& Ochs, S. (1957). Electrical and vascular concomitants of spreading depression. The American Journal of Physiology, 189(1), 159-166. [PMID]

Van Harreveld, A., \& Schadé, J. P. (1962). Changes in the electrical conductivity of cerebral cortex during seizure activity. Experimental Neurology, 5(5), 383-400. [DOI:10.1016/00144886(62)90051-1]

Vongerichten, A. N., Santos, G., Aristovich, K., Avery, J., McEvoy, A., \& Walker, M., et al. (2016). Characterisation and imaging of cortical impedance changes during interictal and ictal activity in the anaesthetised rat. NeuroImage, 124(Pt A), 813-823. [PMID]

Wang, L., Liu, W. B., Yu, X., Xu, C. H., Dong, X. Z., \& Gao, F. (2016). Image monitoring for head phantom of Intracranial Hemorrhage using electrical impedance tomography. 2016 Progress in Electromagnetic Research Symposium (PIERS), 1408-1411. [DOI:10.1109/PIERS.2016.7734663]

Wang, L., Liu, W., Yu, X., Dong, X. Z., \& Gao, F. (2017). Reconstruction and imaging of intracerebral hemorrhage by electrical impedance tomography. Paper presented at: 10th International Congress on Image and Signal Processing, BioMedical Engineering and Informatics (CISP-BMEI), Shanghai, China, 14-16 October 2017. [DOI:10.1109/CISP-BMEI.2017.8302181]

Wang, L., Sun, Y., Xu, X., Dong, X., \& Gao, F. (2017). Real-time imaging of epileptic seizures in rats using electrical impedance tomography. Neuroreport, 28(11), 689-693. [PMID]

Wanjun, S., Fusheng, Y., Wei, Z., Hongyi, Z., Feng, F., \& Xuetao, S., et al. (2008). Image monitoring for an intraperitoneal bleeding model of pigs using electrical impedance tomography. Physiological Measurement, 29(2), 217-225. [PMID]

Williams, C. E., Gunn, A., \& Gluckman, P. D. (1991). Time course of intracellular edema and epileptiform activity following prenatal cerebral ischemia in sheep. Stroke, 22(4), 516-521. [PMID]

Witkowska-Wrobel, A., Aristovich, K., Faulkner, M., Avery, J., \& Holder, D. (2018). Feasibility of imaging epileptic seizure onset with EIT and depth electrodes. NeuroImage, 173, 311-321. [PMID] 
Xu, C. H., Wang, L., Shi, X. T., You, F. S., Fu, F., \& Liu, R. G., et al. (2010). Real-time imaging and detection of intracranial haemorrhage by electrical impedance tomography in a piglet model. The Journal of international Medical Research, 38(5), 1596-1604 [PMID]

Yang, B., Shi, X., Dai, M., Xu, C., You, F., \& Fu, F., et al. (2014). Real-time imaging of cerebral infarction in rabbits using electrical impedance tomography. Journal of International Medical Research, 42(1), 173-183. [PMID]

Yang, L., Liu, W., Chen, R., Zhang, G., Li, W., \& Fu, F., et al. (2017). In Vivo bioimpedance spectroscopy characterization of healthy, hemorrhagic and ischemic rabbit brain within 10 Hz-1 MHz. Sensors (Basel, Switzerland), 17(4), 791. [PMID]

Yang, L., Xu, C., Dai, M., Fu, F., Shi, X., \& Dong, X. (2016). A novel multi-frequency electrical impedance tomography spectral imaging algorithm for early stroke detection. Physiological Measurement, 37(12), 2317-2335. [PMID]

Yang, L., Zhang, G., Song, J., Dai, M., Xu, C., \& Dong, X., et al. (2016). Ex-vivo characterization of bioimpedance spectroscopy of normal, ischemic and hemorrhagic rabbit brain tissue at frequencies from $10 \mathrm{~Hz}$ to $1 \mathrm{MHz}$. Sensors (Basel, Switzerland), 16(11), 1942. [PMID]

Zou, Y., \& Guo, Z. (2003). A review of electrical impedance techniques for breast cancer detection. Medical Engineering $\mathcal{E}$ Physics, 25(2), 79-90. [DOI:10.1016/S1350-4533(02)00194-7] 
This Page Intentionally Left Blank 